\title{
Psychiatric hospitalisation and the risk of suicide
}

\author{
Matthew Michael Large and Nav Kapur
}

\section{Summary}

The association between current or recent psychiatric hospitalisation and increased suicide risk is well described. This relationship is generally assumed to be due to the selection of people at increased risk of suicide for psychiatric admission and subsequent failure of protection from suicide once admitted. Here, Matthew Large and Nav Kapur debate whether or not admission to hospital also selects for vulnerability to certain harmful aspects of hospitalisation and whether the increased rate of suicide in current and recently discharged psychiatric patients is, in fact, due to psychiatric hospitalisation itself.

\section{Declaration of interest}

M.M.L. has provided expert testimony in legal proceedings following in-patient suicide. N.K. sits on the Department of Health (England) National Suicide Prevention Strategy Advisory group.

\section{Copyright and usage}

(c) The Royal College of Psychiatrists 2018

\section{For}

Hospitalisation has long been a central element of psychiatric care. A psychiatric admission allows a more thorough assessment, more intensive nursing, it restricts access to some suicide methods and facilitates a range of treatments that are hard to institute in the community. Hospitals tend to admit people with severe mental illness or overwhelming distress, and it is unfortunate but unsurprising that suicide rates among current and recently discharged psychiatric in-patients are extremely high. The prevailing view is that the selection of high-risk people and a tragic failure of protection explain the strong association between psychiatric hospitalisation and suicide. I will be arguing that this is unlikely to be the whole story, and that psychiatric hospitalisation might actually be increasing suicide.

Psychiatric hospitalisation undoubtedly saves some people's lives. However, anyone who has worked in a psychiatric hospital will recall patients who have been highly distressed or traumatised by aspects of hospitalisation. The peer-reviewed literature provides ample evidence that hospital treatment can be perceived as humiliating, ${ }^{1}$ stigmatising, ${ }^{2}$ coercive ${ }^{3}$ and traumatic. ${ }^{4}$ Hospitalisation often results in a loss of social support and social role, ${ }^{5}$ and violent victimisation is frighteningly common. ${ }^{6}$ In-patients who are already vulnerable are likely to be particularly susceptible to these factors, which are known to be associated with suicide.

The 'yes case' starts with the assumption that it is simply implausible that adverse experiences in hospital never precipitate suicide. ${ }^{8-10}$ However, the yes case has a much higher burden of proof; it has to show, on the balance of probability, that hospitalisation increases the overall risk of suicide. Specifically, it needs to succeed in arguing that hospitalisation causes so many suicides, and/or that it protects so few, that the number of suicides caused exceeds the number of lives saved. The conventional wisdom that psychiatric hospitals are places of asylum along with the natural human tendency to overlook the ill effects of our own actions makes this task more difficult.

The winner of this debate will also be the side that best explains what is known about in-patient suicide. Despite the universal practice of admitting some suicidal patients to hospital, there is little or no direct empirical evidence about the effects of hospitalisation on suicide. Two randomised controlled trials have examined the effects of hospitalisation on suicide outcomes. ${ }^{11,12}$ Neither study had the statistical power to examine whether admission increases or reduces suicide.

My colleagues and I started to worry about a possible causal relationship between hospitalisation and suicide while performing a meta-analysis of rates of in-patient suicide. ${ }^{13}$ We examined studies of in-patient suicide published over 60 years and found that 1 in 676 (95\% CI 604-755) admissions ended in a suicide; a rate of 147 suicides per 100000 patient years. What struck us most was the immense variation in the reported suicide rates. One English study reported a rate of 9 per 100000 patient years, ${ }^{14}$ whereas an Australian study reported the extraordinary rate of 10490 suicides per 100000 patient years. ${ }^{15}$ The extent of this variation was not restricted to the outlying studies: the first quartile, median and third quartile were 95, 358 and 808 suicides per 100000 patient years, respectively. The suicide statistics associated with in-patient care after 1999 were even more worrying. In these studies, the suicide rate was 646 per 100000 patient years, a rate that is about than 50 times that of the community. In the later studies, the average length of hospital stay was considerably shorter than in older studies, such that - despite the dramatically increased suicide rate per 100000 patient years - the absolute probability of a suicide during an admission had fallen to 1 in 1222 .

Naturally, some of the association between psychiatric hospitalisation and suicide is confounded by indication. People are often admitted to hospital because of their suicidal thoughts and behaviours, and many will have multiple suicide risk factors; but why should there be such different patient populations between hospitals, or such different abilities in preventing suicide? Could differences in the adverse aspects of hospital care - such as differences in coercion, trauma and humiliation - lead to these greatly differing suicide rates because of different numbers of 'nosocomial' suicides?

The yes case contends that differences in the types of admitted patients and differences in the abilities of hospitals to protect patients are unlikely to account for most of the observed variation in in-patient suicide rates. It is also likely that adverse experiences of the trauma and stigma associated with psychiatric hospital care carry over beyond discharge, and go some way to explaining the extraordinary suicide rates of over 1100 per 100000 patient years in the first few months after discharge. ${ }^{16,17}$

\section{How many suicides does hospitalisation cause?}

There can be little doubt that patient selection and subsequent failure of protection go some way to explaining high and highly variable inpatient suicide rates. ${ }^{10}$ However, it is also the case that everything we know about the rate, variation and lack of predictability of in-patient suicide could be explained by a greatly varying proportion of patients (with a mean of about 1 in 1000) being tipped to suicide by the varying degrees of trauma, stigma, despair and loss of social role experienced across varying hospital settings. However, in addition 
to simply being plausible, a casual association between psychiatric hospitalisation and suicide is supported by most of the additional criteria considered by Austin Bradford Hill to be helpful in distinguishing causal from non-causal associations. ${ }^{10,18}$

Few would doubt that trauma, stigma and loss of social role are plausible causal mechanisms between hospitalisation and suicide. The strength of the association between hospitalisation and suicide is undoubted; it is greater than the association between smoking and lung cancer. According to Hill, strong associations are more likely to be causal than weak associations. This strong association is also consistent in that it has been demonstrated by studies conducted in different times and places. The increased suicide risk very soon after discharge along with the observation that many of those who die by suicide as in-patients were not assessed as suicidal at the point of admission ${ }^{19}$ both point to a temporal relationship between hospitalisation and suicide. The observation that patients with longer admissions or a larger number of admissions are at a greater risk of suicide ${ }^{19}$ supports a dose-response relationship (although this might also be explained by such patients having an increased time at risk). Finally, rates of suicide are analogously high in other custodial settings such as prisons and immigration detention centres.

\section{How many lives are saved by hospitalisation?}

Hospitalisation might not prevent as many suicides as we would like to imagine. It is widely recognised that few high-risk patients actually die by suicide and it should not be assumed that the lack of suicide by the vast majority of patients is because hospitalisation is so helpful. First, the most evidence-based methods for reducing in-patient suicide are intermittent nursing observations and the elimination of hanging points. However, these methods can only reduce onward suicides, whereas the majority of in-patient suicides occur on approved or unapproved leave. Second, it is hard to argue that hospitals prevent suicide when hospitals so rarely implement any therapies that are specifically designed to reduce suicide. Third, the actual rates of in-patient suicide make it hard to argue that hospitalisation is very strongly protective. Finally, the frighteningly high rate of suicide after discharge from psychiatric hospitals all but proves that any protective effect of hospitalisation is short lived.

Matthew Large

\section{Against}

How do we manage someone who is in acute distress and wishes to take their own life? By assessing their needs and deciding on the best course of treatment and then sometimes, almost as a last resort, admitting them to an in-patient bed. This is one of the most intensive treatment options available in psychiatry and clinicians all know situations when it has been life saving. Of course, variations in the quality of treatment are common across health settings and there may be in-patient units that provide less-than-ideal care. But, equally, there are excellent facilities that are therapeutic in every sense. I agree that psychiatric in-patient admission may not be suitable for everyone but that is not the focus of this debate. The assertion that in-patient care increases rather than decreases suicide risk is simply not credible. Psychiatric admission does not kill more people than it cures.

\section{Correlation $\boldsymbol{v}$. causation}

One of Professor Large's central arguments is that the high rate of suicide among psychiatric in-patients indicates that admission causes suicide. This is a flawed conclusion; correlation does not equal causation. We carried out a national study in England where we examined the role of staffing in relation to suicide rates ${ }^{20}$ and found a moderately strong positive correlation between concentration of senior psychiatrists in a mental health service and the suicide rate. Does this mean that psychiatrists cause suicide? Of course not, it is simply that the services with the highest level of patient need and patient morbidity are likely to have the highest staffing levels. Another study from Denmark showed a high relative risk of suicide among patients admitted to hospital but also a high rate among patients receiving out-patient treatment or just medication as compared with those receiving no care. ${ }^{21}$ If we follow Professor Large's line of reasoning, the safest option is to leave patients untreated! These findings do not reflect the relative safety of different types of care. It is a selection effect whereby the most unwell patients with the most complex needs are getting the most intensive levels of treatment; in other words, 'confounding by indication'.

How do we know that this is the case? Is there any research evidence to back this up? We carried out a study of over 38000 people with self-harm and investigated how the management they received in hospital related to the subsequent risk of mortality. ${ }^{22}$ The study found that patients who were admitted to a psychiatric bed were over twice as likely to die from any cause over the next 12 months than patients who were not admitted. Does this mean that psychiatric admission was causing these deaths? No, it does not: when the results were adjusted for patient characteristics, i.e. when potential confounders were taken into account, the risk reduced and was no longer elevated. Indeed, for some high-risk groups of people (e.g. men aged over 65 and/or those with a previous history of suicidal behaviour), psychiatric admission was actually associated with a lower risk of death. Admission to a psychiatric bed may actually have been saving lives.

Professor Large is right in that there are no randomised controlled trials of in-patient admission with suicide as an outcome; but the ethical, moral and practical challenges inherent in such studies are likely to be insurmountable. He is also right in that not all therapeutic options are suitable for all patients. We as clinicians constantly make decisions, ideally in collaboration with our patients, about what treatments might be most suitable. For some patients admission is that best option.

Professor Large says that to win the debate he has to show 'that hospitalisation causes so many suicides, and/or that it protects so few, that the number of suicides caused exceeds the number of lives saved.' His paragraphs on how many lives hospitalisation saves or how many suicide deaths it causes are a little short on numbers. He fails to satisfy his own debate criteria.

\section{Trends in in-patient suicide}

Temporal trends in in-patient suicide need to be considered, particularly with respect to what these trends reveal about safety. In the UK, in common with much of the Western world, the number of psychiatric in-patient beds has shrunk considerably over time, with an increasing emphasis on community care. Therefore, thresholds for admission have changed and the patients who currently get admitted are - on the whole - more unwell than in the past. This might be expected to push up suicide rates. However, suicide rates in the general population have fallen and there is a focus on safety within in-patient units. For example, ligature points have been removed and people are prevented from leaving the ward without staff permission. With these contradictory pressures on suicide rates, the question is which way have they gone? In fact, in-patient suicide rates have gone down substantially. ${ }^{23}$ So it would appear that not only are psychiatric in-patient units (at least in the UK) safe, but they may be getting safer. 
In recent years, care has shifted to community services that are alternatives to in-patient admission - so-called hospital at home or home-treatment teams. In England we investigated the number of deaths under the care of crisis resolution home treatment teams $(\mathrm{CRHT}){ }^{24}$ There has been a marked increase in this type of care in recent years and there are currently three times the number of suicide deaths under CRHT compared with in-patient settings. To an extent, this is what we would expect given the huge increase in the number of people being treated by home treatment teams. Rates are more difficult to calculate reliably, but when we attempt to do this the findings are surprising. The suicide rate under CRHT is almost double the rate under in-patient care. It is difficult to argue that psychiatric admission is killing more people than it cures when the in-patient setting appears to be associated with half the suicide rate.

Nav Kapur

\section{For: rebuttal}

In his first paragraph, Professor Kapur states that the yes case is 'simply not credible'. I obviously disagree. Professor Kapur has only been able to foreclose on such an important issue so early because he has not really addressed the nub of the yes case. Although the very high rates of in-patient suicide are undoubtedly important, the yes case did not mistake correlation for causation. I accept that hospitalisation selects for people who are at high risk and have stated this clearly here and in earlier papers. ${ }^{8,10}$ However, hospitalisation also selects for vulnerable people, people who might not be able to cope with environments characterised by Professor Kapur as being less than ideal. The starting point of the yes case is that both the extremely high rates of suicide in psychiatric hospitals and their huge heterogeneity need to be explained. The heterogeneity cannot be explained by patient characteristics: people do not differ that much. A more likely explanation is the variation in the quality of care so frankly admitted by Professor Kapur. In fact, the only explanation offered by Professor Kapur is almost identical to mine: 'variations in the quality of treatment are common across health settings and there may be in-patient units which provide less-than-ideal care.' The only difference between the yes and no case on this point is Professor Kapur's use of the euphemism 'less than ideal'. Any experienced clinician will know of some psychiatric wards with high rates of violence, physical restraint and seclusion; wards with such little therapeutic value that they would never want their family or friends admitted to them.

Professor Kapur asserts that suicide rates have gone down. It is true that in-patient suicide rates have fallen in recent years in the UK. However, the bigger picture, based on worldwide data over much longer periods of time, ${ }^{13}$ is that rates of suicide in psychiatric facilities have increased. The most obvious explanation for this is a selection effect, because only patients who are more severely ill can be accommodated in a shrinking number of beds. There is little doubt that the increased acuity of psychiatric wards has made them more confronting and more traumatic. It is logical that this more frightening and traumatising environment would therefore be more likely to precipitate suicide.

\section{How should we decide if hospitalisation increases or decreases suicide risk?}

I acknowledge that the yes case is not proven numerically. It is simply not known how many patients are protected and how many die because of hospitalisation. For the purpose of this debate, the reader has to make a judgement on the basis of the available evidence and the likely implications for patient care. Austin
Bradford Hill believed that, in deciding whether an association is causal, we should not simply consider the facts but should also consider the consequences of our beliefs. ${ }^{18}$ He acknowledged that this was not strictly scientific. Nowadays, we are familiar with this sort of thinking; for example, one does not have to totally accept that humans are the cause of climate change - or even that climate change exists - to believe that the modest costs of carbon mitigation outweigh the potentially catastrophic consequences of doing nothing. Suicide is so catastrophic that we should think about the costs and consequences of accepting or rejecting the arguments of the yes and no case.

Even if it is likely that a minority of in-patient suicides are a direct result of psychiatric hospitalisation, we should still act to improve hospital care. If the judgement is that hospitalisation does not prevent many suicides and if it is likely that hospitalisation causes more suicide that it prevents, then there are compelling reasons to improve both the quality of hospital care and the access to community care. The advantage of a clear decision for the yes case is that it leaves no room for excuses for less traumatic and less stigmatising psychiatric care.

Matthew Large

\section{Against: rebuttal}

I am very glad that Professor Large accepts that the main reason for the high rate of suicide among people admitted to psychiatric care is a selection effect. Simply put, patients at the greatest risk of suicide are being admitted to hospital. His other point is about variation. Variation in outcome is intriguing and a potentially powerful way of investigating patient safety. But the argument that the differential suicide rates prove that in-patient admission is a bad thing is not convincing. What is the evidence that (even in the units with higher rates of suicide) admitting people is doing more harm than good? Yes, rates of in-patient suicide have gone up in some countries and, yes, the most likely contributory factor is the reduced number of in-patient beds, meaning that patients may be more unwell before being admitted than they were in the past. But is there any evidence that this has made psychiatric wards less safe, to the extent that in-patient admission now causes more deaths than it prevents? I do not think that there is.

\section{Evidence for a dose response}

If we imagine that Professor Large's assertion that psychiatric admission is causing suicide is true, we might expect to see a dose-response relationship. That is, the highest suicide risk will be in people who have been most exposed to the 'toxic' stimulus, i.e. those with the longest lengths of admission. In fact, when we look at the best studies examining suicide risk by time period, we find that suicide risk is highest early on in the admission. ${ }^{25}$ Rates of suicide decrease the longer someone has been in hospital care, meaning there is no evidence for a dose-response relationship. The high rates of suicide early on probably reflect the acute risk of suicide and the severity of illness when people first get admitted.

\section{Evidence that discharge from hospital is protective}

If psychiatric in-patient admission led to suicide, we would expect to see a lowering of the suicide rate as soon as patients were discharged from hospital, i.e. removing people from this toxic environment would be protective. However, research worldwide suggests the opposite: there is a huge jump in suicide rate in the immediate post-discharge period. Data from the National Confidential Inquiry into Suicide and Homicide ${ }^{26}$ in the UK suggest that the highest risk is in the first week after discharge, and people are 
actually at risk as soon as they leave hospital. So what may be happening is the in-patient environment is protective and that protection ceases to be in place when patients return to the community. This hypothesis is consistent with what patients tell us in qualitative studies. ${ }^{27}$ Also relevant here (and to my point about dose response) is the evidence that people who die by suicide in the post-discharge period are more likely to have had short in-patient admissions. ${ }^{28}$

Nav Kapur

\section{For: conclusion}

Professor Kapur puts a lot of weight on what he would term a lack of evidence for a dose-response relationship. Yet such a dose relationship is not as absent as he asserts: there strong evidence that patients who have had more admissions and those that have had longer admissions are at an increased risk of in-patient suicide. ${ }^{19}$

The peak in suicides shortly after discharge presents a more complex issue. Professor Kapur cites English evidence that post-discharge suicide is associated with shorter admissions, but meta-analysis suggests this is not generally true. ${ }^{29}$ Irrespective of the length of stay, it is likely that some patients bide their time until the protections of hospital are removed by discharge. Further, some vulnerable patients are likely so affected by stigma and so traumatised that they suicide at an early opportunity after discharge.

It seems that the yes and no cases reasonably disagree on the central point of causation. This leaves readers to make up their own minds about the evidence with the interests of patients in mind. However, what we can agree about is likely more certain and just as important. There can be few arguments on the need to find better ways of managing more patients in their homes, especially those patients who dislike hospital treatment. We agree that when hospitalisation is unavoidable, as it often will be, that we need to maximise the therapeutic aspects of in-patient psychiatric care and develop suicide-specific prevention strategies. I am sure we agree that we should ensure that patients maintain social roles and social supports during their stay and do whatever possible to make in-patient care less stigmatising and less traumatic.

Matthew Large

\section{Against: conclusion}

\section{The problems with in-patient care}

It is clear that Professor Large and I are starting to agree. Of course in-patient units may not be perfect. Well-documented problems include overcrowding, violence, poor staff morale, and alcohol and substance misuse. Our own work on in-patient observation suggested that interventions were often carried out by the most junior staff. Sometimes observation was seen as an end in itself rather than as a skilled therapeutic intervention. ${ }^{30}$ Patient preference is a major consideration when thinking about treatment setting and of course we need to work hard to ensure that community alternatives to inpatient care are as safe and effective as possible. ${ }^{24}$

\section{Making in-patient care safer}

All treatments may have their side effects, but the assertion that admitting distressed and unwell patients to psychiatric beds is more harmful than helpful is simply not borne out by the evidence. Many of the studies I have drawn on are UK based but there is no reason to assume the findings are not generalisable, especially since some of the most important safety issues in in-patient mental healthcare are universal.

We need to move on and think about how we can make inpatient wards as good as possible. This is something that has received some attention internationally but it needs ongoing discussion. Reports in the $\mathrm{UK}^{26,31}$ have suggested a number of principles: The philosophy of care should be holistic and patient centred. Staff need to be adequately trained and well supervised. Care pathways should to be clear and services must be accessible. We need calming and welcoming in-patient units which are conducive to recovery. The environment should be safe and free from hazards such as ready access to means of suicide (e.g. ligature points). Monitoring the routine outcomes of in-patient admission could help us to ensure that we are providing the best possible care. The practice of admitting patients many miles from their own home because of bed shortages should end. Measures like these taken together will help us to improve mental health in-patient safety and save more lives.

Nav Kapur

For: Matthew Michael Large, BSC (Med), MB BS, DMedSCi, FRANZCP, The Euroa Centre, The Prince of Wales Hospitals, Barker St, Randwick, 2031, NSW, Australia. Email: mmclarge@gmail.com; Against: Nav Kapur, MBChB, MMedSc, FRCPsych, MD, Centre for Suicide Prevention, Centre for Mental Health and Safety, Division of Psychology and Mental Health, University of Manchester and Greater Manchester Mental Health National Health Service Foundation Trust, M13 9PL, UK. Email: nav.kapur@manchester.ac.uk.

First received 12 Jan 2018, accepted 15 Jan 2018

\section{Acknowledgments}

This paper is based on a debate between M.M.L. and N.K. which took place at the Royal Australian and New Zealand College of Psychiatrists 2017 Congress.

\section{References}

1 Svindseth MF, Dahl AA, Hatling T. Patients' experience of humiliation in the admission process to acute psychiatric wards. Nord J Psychiatry 2007; 61: 47-53.

2 Moses T. Stigma apprehension among adolescents discharged from brief psychiatric hospitalization. J Nerv Ment Dis 2011; 199: 778-89.

3 Sheehan KA, Burns T. Perceived coercion and the therapeutic relationship: a neglected association? Psychiatr Serv 2011; 62: 471-6.

4 Paksarian D, Mojtabai R, Kotov R, Cullen B, Nugent KL, Bromet EJ. Perceived trauma during hospitalization and treatment participation among individuals with psychotic disorders. Psychiatr Serv 2014; 65: 266-9.

5 Kinard EM, Klerman LV. Changes in life style following mental hospitalization. J Nerv Ment Dis 1980; 168: 666-72.

6 Iozzino L, Ferrari C, Large M, Nielssen O, de Girolamo G. Prevalence and risk factors of violence by psychiatric acute inpatients: a systematic review and meta-analysis. PLOS ONE 2015; 10: e0128536.

7 Gallop R, McCay E, Guha M, Kahn P. The experience of hospitalization and restraint of women who have a history of childhood sexual abuse. Health Care Women Int 1999; 20: 401-16.

8 Large M, Ryan C, Walsh G, Stein-Parbury J, Patfield M. Nosocomial suicide. Australas Psychiatry 2014; 22: 118-21.

9 Large MM, Ryan CJ. Disturbing findings about the risk of suicide and psychiatric hospitals. Soc Psychiatry Psychiatr Epidemiol 2014; 49: 1353-5.

10 Large MM, Chung DT, Davidson M, Weiser M, Ryan CJ. In-patient suicide: selection of people at risk, failure of protection and the possibility of causation. BJPsych Open 2017; 3: 102-5.

11 Waterhouse J, Platt S. General hospital admission in the management of parasuicide. A randomised controlled trial. Br J Psychiatry 1990; 156: 236-42.

12 van der Sande R, van Rooijen L, Buskens E, Allart E, Hawton K, van der Graaf $Y$, et al. Intensive in-patient and community intervention versus routine care after attempted suicide. A randomised controlled intervention study. Br J Psychiatry 1997; 171: 35-41.

13 Walsh G, Sara G, Ryan CJ, Large M. Meta-analysis of suicide rates among psychiatric in-patients. Acta Psychiatr Scand 2015; 131: 174-84.

14 Copas JB, Robin A. Suicide in psychiatric in-patients. Br J Psychiatry 1982; 141: 503-11. 
15 Bassett D, Tsourtos G. Inpatient suicide in a general hospital psychiatric unit. A consequence of inadequate resources? Gen Hosp Psychiatry 1993; 15: 301-6.

16 Chung DT, Ryan CJ, Large MM. Commentary: adverse experiences in psychiatric hospitals might be the cause of some postdischarge suicides. Bull Menninger Clin 2016; 80: 371-5

17 Chung DT, Ryan CJ, Hadzi-Pavlovic D, Singh SP, Stanton C, Large MM. Suicide rates after discharge from psychiatric facilities: a systematic review and metaanalysis. JAMA Psychiatry 2017; 74: 694-702.

18 Hill AB. The environment and disease: association or causation? Proc $R$ SOC Med 1965; 58: 295-300.

19 Large M, Smith G, Sharma S, Nielssen O, Singh SP. Systematic review and metaanalysis of the clinical factors associated with the suicide of psychiatric inpatients. Acta Psychiatr Scand 2011; 124: 18-29.

20 Kapur N, Ibrahim S, while D, Baird A, Rodway C, Hunt IM, et al. Mental health service changes, organisational factors, and patient suicide in England in 1997-2012: a before-and-after study. Lancet Psychiatry 2016; 3: 526-34.

21 Hjorthoj CR, Madsen T, Agerbo E, Nordentoft M. Risk of suicide according to level of psychiatric treatment: a nationwide nested case-control study. SOC Psychiatry Psychiatr Epidemiol 2014; 49: 1357-65

22 Kapur N, Steeg S, Turnbull P, Webb R, Bergen H, Hawton K, et al. Hospital management of suicidal behaviour and subsequent mortality: a prospective cohort study. Lancet Psychiatry 2015; 2: 809-16.

23 Kapur N, Hunt IM, Windfuhr K, Rodway C, Webb R, Rahman MS, et al. Psychiatric in-patient care and suicide in England, 1997 to 2008: a longitudinal study. Psychol Med 2013; 43: 61-71.
24 Hunt IM, Rahman MS, While D, Windfuhr K, Shaw J, Appleby L, et al. Safety of patients under the care of crisis resolution home treatment services in England: a retrospective analysis of suicide trends from 2003 to 2011. Lancet Psychiatry 2014; 1: 135-41.

25 Qin P, Nordentoft M. Suicide risk in relation to psychiatric hospitalization: evidence based on longitudinal registers. Arch Gen Psychiatry 2005; 62: 427-32.

26 National Confidential Inquiry into Suicide and Homicide (NCISH). Making Mental Health Care Safer. Annual Report and 20-year Review.University of Manchester, 2016.

27 Owen-Smith A, Bennewith O, Donovan J, Evans J, Hawton K, Kapur N, et al. 'When you're in the hospital, you're in a sort of bubble.' Understanding the high risk of self-harm and suicide following psychiatric discharge: a qualitative study. Crisis 2014; 35: 154-60.

28 Bickley H, Hunt IM, Windfuhr K, Shaw J, Appleby L, Kapur N. Suicide within two weeks of discharge from psychiatric inpatient care: a case-control study. Psychr Serv 2013; 64: 653-9.

29 Large M, Sharma S, Cannon E, Ryan C, Nielssen O. Risk factors for suicide within a year of discharge from psychiatric hospital: a systematic meta-analysis. Aust N Z J Psychiatry 2011; 45: 619-28.

30 National Confidential Inquiry into Suicide and Homicide (NCISH). In-patient Suicide Under Observation. University of Manchester, 2015.

31 Crisp N, Smith C, Nicholson K (eds). Old Problems, New Solutions: Improving Acute Psychiatric Care for Adults in England (Final Report). The Commission on Acute Adult Psychiatric Care, 2016. Available at: http://www.rcpsych.ac.uk pdf/Old Problems_New Solutions_CAAPC Report England.pdf (accessed 1 Feb 2018). 